\title{
16. On the Periods of Enriques Surfaces. II
}

\author{
By Eiji HORIKAWA \\ University of Tokyo
}

(Communicated by Kunihiko KodaIra, M. J. A., April 12, 1977)

This is a continuation of [4], and here we report on our result on the image of the period map for Enriques surfaces.

Let $S$ be an Enriques surface defined over the field of complex numbers. Then there corresponds to $S$ a point $\lambda(S)$, called the period of $S$, which is in the period space $D / \Gamma$. First we recall the construction of $D$ and $\Gamma$. Let $T$ be the universal covering of $S$. Then $T$ is a $K 3$ surface, and hence the homology group $H_{2}(T, Z)$, given with the intersection product, is isomorphic to a unique even unimodular euclidean lattice $\Lambda$ of signature $(3,19)$. Moreover, if we associate the involution $\tau$ induced by the covering transformation, the pair $\left(H_{2}(T, Z), \tau\right)$ is isomorphic to a standard pair $(\Lambda, \rho)$ (see $[4], \S 3$ ). Let $\Lambda(-1)$ denote the (-1)-eigenspace of $\rho$. Then $D$ consists of non-zero linear maps $\omega: \Lambda(-1) \rightarrow C$, modulo multiplications by constants, which satisfy the Riemann bilinear relations

$$
\omega \cdot \omega=0, \quad \omega \cdot \bar{\omega}>0,
$$

the product being induced by that on $\Lambda(-1)$. On the other hand, $\Gamma$ is the group of those automorphisms of $\Lambda(-1)$ which are the restrictions of the automorphisms of $\Lambda$ commuting with $\rho$.

An element $e$ of $\Lambda(-1)$ is called a root if it satisfies $e^{2}=-2$. From the explicit description of $\Lambda(-1)$ in [4], we infer that such elements exist. If $e$ is a root, we define a hypersurface $H_{e}$ of $D$ by the condition $\omega(e)=0$. We shall use $H_{e} / \Gamma$ to denote $H_{e} \Gamma / \Gamma$.

Main Theorem. There exists only a finite number of $\Gamma$-equivalence classes of the roots $e$ in $\Lambda(-1)$, and if $\lambda$ is a point of $D / \Gamma$ outside of the union of the hypersurfaces $H_{e} / \Gamma$, then $\lambda$ is the period of an Enriques surface $S$, which is uniquely determined by $\lambda$. Moreover, any point of $H_{e} / \Gamma$ is not the period of an Enriques surface.

The basic idea of the proof is that of [3].

First, by the construction in [4], each Enriques surface $S$ is birationally equivalent to a double covering of $\boldsymbol{P}^{1} \times \boldsymbol{P}^{1}$. We take a system of 2-way homogeneous coordinates $\left(Y_{1}, Y_{2} ; Z_{1}, Z_{2}\right)$ and fix the projection onto the second factor. Then the branch locus of the covering consists of the two fibres $\Gamma_{i}$ defined by $Z_{i}=0, i=1,2$, and a curve $B_{E}^{0}$ of bidegree $(4,4)$, which has two 2 -fold double points at $P_{i}$ on $\Gamma_{i}$, having the contact of order 4 with $\Gamma_{i}$ at $P_{i}, i=1,2$. An Enriques surface $S$, with an elliptic 
pencil being specified, is said to be of special type, if $P_{1}$ and $P_{2}$ are on a section $Y_{1}=\beta Y_{2}$ for some constant $\beta$ (possibly $\infty$ ). Suppose this is not the case. Then we may assume that $P_{i}$ is given by $Y_{i}=Z_{i}=0$, $i=1,2$. Hence $B_{E}^{0}$ is defined by a linear combination of the monomials

$$
Y_{1}^{i} Y_{2}^{4-i} Z_{1}^{j} Z_{2}^{4-j}, \quad 4 \leqq i+2 j \leqq 8 .
$$

In order to obtain a model for the universal covering $T$, we consider the double covering $\pi: \boldsymbol{P}^{1} \rightarrow \boldsymbol{P}^{1}$ branched at $Z_{1}=0$ and $Z_{2}=0$. Pulling $B_{E}^{0}$ back by $\pi$, and applying two elementary transformations, we see that $T$ is birationally equivalent to a double covering of $\boldsymbol{P}^{1} \times \boldsymbol{P}^{1}$, whose branch locus $B$ is of bidegree $(4,4)$, and is defined by a linear combination of the monomials

(1) $\quad Y_{1}^{i} Y_{2}^{4-i} Z_{1}^{j} Z_{2}^{4-j}, \quad i+j \equiv 0 \quad \bmod 2$.

The covering transformation is induced by

(2) $\quad I:\left(Y_{1}, Y_{2} ; Z_{1}, Z_{2}\right) \rightarrow\left(-Y_{1}, Y_{2} ;-Z_{1}, Z_{2}\right)$,

and the interchange of the sheets of $T \rightarrow \boldsymbol{P}^{1} \times \boldsymbol{P}^{1}$.

Next we consider Baily-Borel's compactification $(D / \Gamma)^{*}$ of $D / \Gamma$. Suppose that the branch locus $B$ degenerates into a singular one. Then we take a 1-parameter family of the divisors defined by (1), whose generic member is non-singular. This determines a point in $(D / \Gamma)^{*}$, which can be thought of as the period corresponding to $B$. Note that this point may depend on the choice of the 1-parameter family.

If $B$ passes through a fixed point of $I$ defined by (2), say $Y_{1}=Z_{1}=0$, then the corresponding double covering $T$ is still birationally equivalent to a $K 3$ surface. $T$ has a double point over $Y_{1}=Z_{1}=0$, and this is a fixed point of the involution $\iota$ induced by $I$ and the interchange of the sheets. Therefore the quotient space $T / \iota$ is not an Enriques surface, but a rational surface with a rational quadruple point. In this case the period is in $H_{e} / \Gamma$ for some root $e$.

If $B$ has infinitely near triple points, a quadruple point, or a double component, then the corresponding $K 3$ surface degenerates into a union of two rational surfaces intersecting along an elliptic curve (in "generic" cases). In this case the corresponding period is in the boundary of $(D / \Gamma)^{*}$. If $B$ does not pass through the fixed points of $I$, then the corresponding degeneration of Enriques surface is a rational surface with a double curve along an elliptic curve. If $B$ passes through a fixed point of $I$, it corresponds to a union of six rational surfaces, which consists of two rational surfaces $S_{1}, S_{2}$ intersecting transversally along a rational curve, and four $\boldsymbol{P}^{2}$ 's, each of which intersects $S_{1}$ and $S_{2}$ like three coordinate planes in $C^{3}$.

In these two cases, the period does not depend on the choice of the 1-parameter family which we use. This fact follows from the extension theorem of Borel [2] and others. The same extension theorem allows us to restrict our consideration to generic cases. 
Finally suppose that $B$ has a triple or quadruple component. Then that component is of the form $Y_{1} Z_{2}-\alpha Y_{2} Z_{1}=0$ with some constant $\alpha$. These cases can be reduced to the cases of lower multiplicities by blowing up along the multiple component (cf. [3], §§10-12. But the situation is more transparent here than it was there). In the case of triple components, the corresponding periods lie in the closure of the union of the hypersurfaces $H_{e} / \Gamma$. The case of quadruple components corresponds to Enriques surfaces of special type and their degenerations.

\section{References}

[1] Baily, W. L., Jr., and Borel, A.: Compactification of arithmetic quotients of bounded symmetric domains. Ann. of Math., 84, 442-528 (1966).

[2] Borel, A.: Some metric properties of arithmetic quotients of symmetric spaces and an extension theorem. J. Diff. Geometry, 6, 543-560 (1972).

[3] Horikawa, E.: Surjectivity of the period map of $K 3$ surfaces of degree 2 (to appear in Math. Ann.).

[4] - On the periods of Enriques surfaces. I. Proc. Japan Acad., 53, 124-127 (1977). 